\title{
Structure and function of aerotolerant, multiple- turnover THI4 thiazole synthases
}

\author{
Jaya Joshi ${ }^{1, \star}$, Qiang Li ${ }^{2, \star}$, Jorge D. García-García ${ }^{1}$, Bryan J. Leong ${ }^{1}$, You Hư ${ }^{2}$, Steven D. Bruner ${ }^{2}$ and \\ (ㄱ) Andrew D. Hanson ${ }^{1}$ \\ ${ }^{1}$ Department of Horticultural Sciences, University of Florida, Gainesville, FL, U.S.A.; ${ }^{2}$ Department of Chemistry, University of Florida, Gainesville, FL, U.S.A.
}

Correspondence: Andrew D. Hanson (adha@ufl.edu)

\section{OPEN ACCESS}

*These authors contributed equally to this work.

Received: 2 August 2021 Revised: 18 August 2021 Accepted: 19 August 2021

Accepted Manuscript online: 19 August 2021

Version of Record published: 7 September 2021
Plant and fungal THI4 thiazole synthases produce the thiamin thiazole moiety in aerobic conditions via a single-turnover suicide reaction that uses an active-site Cys residue as sulfur donor. Multiple-turnover (i.e. catalytic) THI4s lacking an active-site Cys (non-Cys THI4s) that use sulfide as sulfur donor have been biochemically characterized -- but only from archaeal methanogens that are anaerobic, $\mathrm{O}_{2}$-sensitive hyperthermophiles from sulfide-rich habitats. These THI4s prefer iron as cofactor. A survey of prokaryote genomes uncovered non-Cys THI4s in aerobic mesophiles from sulfide-poor habitats, suggesting that multiple-turnover THI4 operation is possible in aerobic, mild, low-sulfide conditions. This was confirmed by testing 23 representative non-Cys THI4s for complementation of an Escherichia coli $\Delta$ thiG thiazole auxotroph in aerobic conditions. Sixteen were clearly active, and more so when intracellular sulfide level was raised by supplying Cys, demonstrating catalytic function in the presence of $\mathrm{O}_{2}$ at mild temperatures and indicating use of sulfide or a sulfide metabolite as sulfur donor. Comparative genomic evidence linked non-Cys THI4s with proteins from families that bind, transport, or metabolize cobalt or other heavy metals. The crystal structure of the aerotolerant bacterial Thermovibrio ammonificans THI4 was determined to probe the molecular basis of aerotolerance. The structure suggested no large deviations compared with the structures of THI4s from $\mathrm{O}_{2}$-sensitive methanogens, but is consistent with an alternative catalytic metal. Together with complementation data, use of cobalt rather than iron was supported. We conclude that catalytic THI4s can indeed operate aerobically and that the metal cofactor inserted is a likely natural determinant of aerotolerance.

\section{Introduction}

Biosynthesis of the adenylated carboxythiazole (ADT) precursor of thiamin is chemically complex and energetically expensive $[1,2]$. Plants, fungi, and some prokaryotes make ADT via the thiazole synthase THI4, a single-turnover suicide enzyme [3-6]. In a reaction requiring iron (yeast) or zinc (Arabidopsis), these THI4s form ADT from NAD, glycine, and a sulfur atom stripped from an activesite Cys residue $[3,5,7,8]$. The sulfur loss converts Cys to dehydroalanine and irreversibly inactivates the enzyme [3,5] (Figure 1). Such THI4s must, therefore, be replaced after just one reaction cycle, and this - plus the high demand for thiazole [9] - makes THI4 one of the shortest-lived proteins in plant leaves $[10,11]$.

Bioenergetic calculations indicate that the cost of THI4 degradation and resynthesis in plants reduces biomass accumulation by $2-4 \%$ [2]. Crop biomass gains of this order could, therefore, result from engineered replacement of a suicide THI4 with a catalytic THI4 that, like most enzymes, mediates thousands of turnovers in its lifetime [12,13]. But do catalytic THI4s that can operate in the mild, aerobic conditions typical of plant cells exist in nature? And if so, what characteristics confer this ability? 


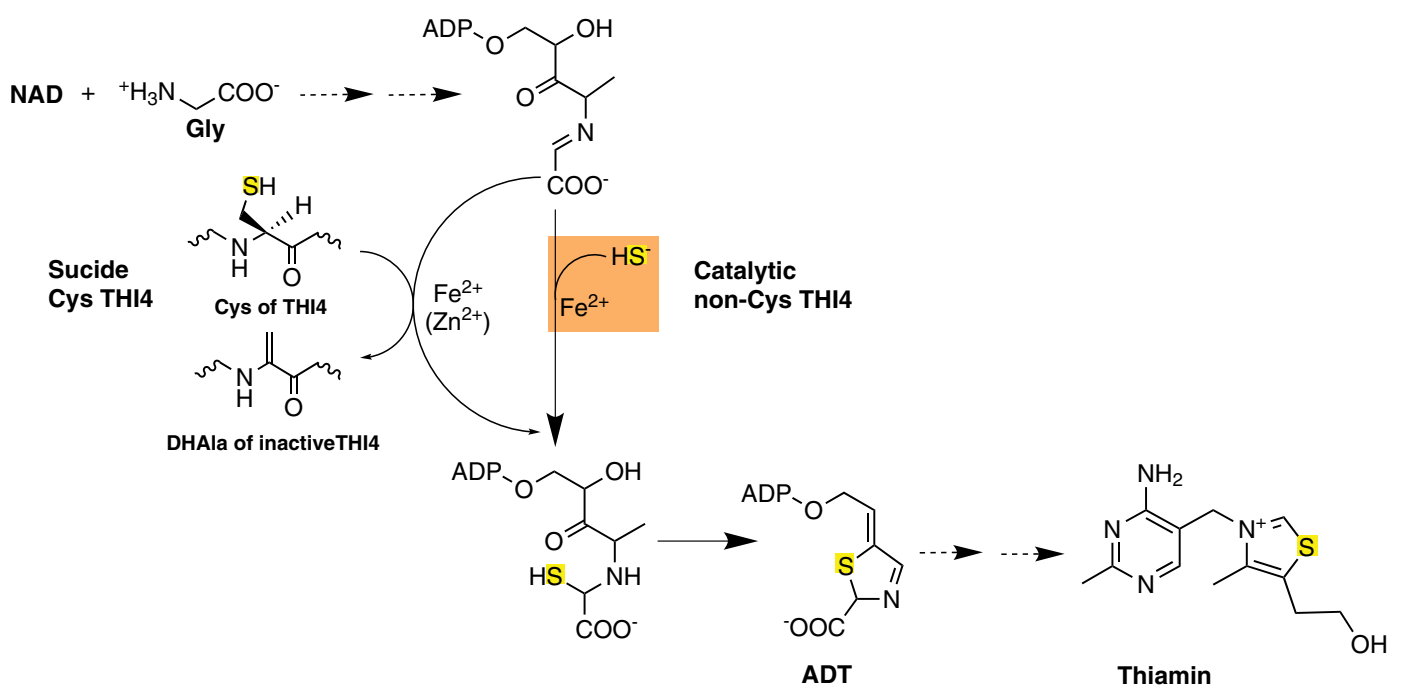

Figure 1. Biosynthesis of the thiazole precursor of thiamin by suicide and catalytic THI4s.

THI4s form the adenylated carboxythiazole (ADT) precursor of thiamin from NAD, glycine, and a sulfur atom that in yeast and plant THI4s comes from an active-site cysteine residue and in methanococcal THI4s comes from sulfide (HS ${ }^{-}$). Sulfur loss from the active-site cysteine leaves a dehydroalanine (DHAla) residue that is not reconverted to cysteine, making yeast and plant THI4s suicide enzymes. In contrast, THI4s that use sulfide mediate multiple turnovers, i.e. are true catalysts.

Catalytic THI4s have been demonstrated biochemically from strictly anaerobic, $\mathrm{O}_{2}$-sensitive, thermophilic methanogens from hydrothermal vents, where sulfide levels are high enough (millimolar) to kill plants and most other organisms [7,14]. These THI4s use sulfide as the sulfur donor, have His in place of Cys in the active site, and prefer iron as cofactor in vitro (Figure 1) [7,14]. Indirect evidence suggests that catalytic, $\mathrm{O}_{2}$-sensitive THI4s may also occur in certain plants [5]. An exploratory survey [15] of prokaryote genomes identified THI4s with no active-site Cys (non-Cys THI4s) in several diverse organisms, and preliminary tests showed that two such THI4s complemented an Escherichia coli thiazole synthase $(\Delta t h i G)$ mutant in aerobic conditions [15]. By indicating that non-CysTHI4s can have at least some activity in mild conditions in the presence of $\mathrm{O}_{2}$, these pilot data prompted further research.

In this work, we deeply surveyed the diversity and genomic contexts of prokaryote non-Cys THI4s and ran complementation assays of thiazole synthase activity on a representative set. In addition, the crystal structure of a THI4 with aerobic complementing activity was determined and this THI4's in vivo metal preference was explored. The results implicated the cofactor metal as a determinant of $\mathrm{O}_{2}$ tolerance.

\section{Materials and methods Chemicals and media}

Chemicals and reagents were from Sigma-Aldrich or Fisher Scientific unless otherwise indicated. MOPS minimal medium was prepared as described [16] except that it was supplemented with the concentrations of micronutrients as specified in [17].

\section{Bioinformatics}

Microbial THI4 sequences were identified in the SEED [18] and UniRef90 [19] databases using Thermovibrio ammonificans THI4 as the query sequence. Comparative genomics analyses were performed using SEED and GenBank resources. Sequence similarity networks (SSNs) were constructed by submitting 199 THI4 sequences to the EFI-EST webtool using the FASTA option [20]. An $E$-value of $10^{-5}$ was used to delimit sequence similarity. A final SSN was generated with an alignment score cutoff set such that each connection (edge) represents $\sim 80 \%$ sequence identity. In this setting, some sequences remained as singletons. Network layouts were created and visualized using Cytoscape 3.4. 


\section{Knockout strain and clone construction}

An E. coli MG1655 $\Delta$ thiG strain was made by recombineering $[15,21]$ using the $\Delta$ thiG cassette from the corresponding Keio collection strain [22]. Selected THI4 genes were recoded for expression in E. coli or yeast and synthesized by GenScript (Piscataway, NJ) or Twist Biosciences (San Francisco, CA). Recoded nucleotide sequences are given in Supplementary Table S1. For E. coli, recoded sequences with an added N-terminal His 6 tag were cloned between the EcoRI and XbaI sites in pBAD24 [23]. For yeast (Saccharomyces cerevisiae), the recoded T. ammonificans THI4 sequence (preceded by the putative yeast THI4 targeting peptide MSATSTATSTSASQLHLNSTPVTHCLSDGG plus a GG linker) or the native yeast THI4 was inserted into the CEN6/ARS4 nuclear plasmid carrying the HIS3 marker and the TDH3 promoter to drive THI4 expression.

\section{THI4 protein expression analysis}

pBAD24 constructs were introduced into the E. coli MG1655 $\Delta$ thiG strain and single colonies were used to inoculate $3 \mathrm{ml}$ of MOPS medium containing $0.2 \%$ (w/v) glycerol, $100 \mathrm{nM}$ thiamin, $100 \mu \mathrm{g} / \mathrm{ml}$ ampicillin, and $50 \mu \mathrm{g} / \mathrm{ml}$ kanamycin. The next day, $25-\mathrm{ml}$ cultures were grown at $37^{\circ} \mathrm{C}$ in MOPS-glycerol medium with $100 \mathrm{nM}$ thiamin supplementation until $\mathrm{OD}_{600 \mathrm{~nm}}$ reached 0.8 . Cells were then induced by adding $0.02 \%(\mathrm{w} / \mathrm{v})$ arabinose and incubated for another $4 \mathrm{~h}$ at $37^{\circ} \mathrm{C}$. Cells were harvested by centrifugation $\left(6000 \mathrm{~g}, 15 \mathrm{~min}, 4^{\circ} \mathrm{C}\right)$, flash-frozen in liquid nitrogen, and stored at $-80^{\circ} \mathrm{C}$. Cell pellets were extracted by sonicating in $1 \mathrm{ml}$ of $100 \mathrm{mM}$ potassium phosphate ( $\mathrm{pH}$ 7.2) containing $2 \mathrm{mM} \beta$-mercaptoethanol, and separated into soluble and insoluble fractions by centrifugation $\left(17000 \mathrm{~g}, 10 \mathrm{~min}, 4^{\circ} \mathrm{C}\right)$. Proteins in the pellet were solubilized by boiling for $5 \mathrm{~min}$ in $0.5 \mathrm{ml}$ of SDS sample buffer. Aliquots $(10 \mu \mathrm{l})$ of the insoluble fraction extract or 10-fold-diluted soluble protein extract were separated by SDS-PAGE on 15\% gels; proteins were detected by Coomassie Blue staining. The identity of the THI4 bands was confirmed by immunoblotting using anti-His ${ }_{6}$ tag antibodies (Thermo Fisher Scientific MA1-21315). The THI4 Coomassie band in soluble and insoluble fractions was quantified using Licor Image Studio Lite software. A 3441-pixel area around the band was used to calculate signal intensity. The method was calibrated using a standard curve for purified recombinant T. ammonificans THI4.

\section{Functional complementation assays in $E$. coli and yeast}

For assays in E. coli, three independent clones of each construct were used to inoculate $3 \mathrm{ml}$ of MOPS medium containing $0.2 \%$ glycerol $(\mathrm{w} / \mathrm{v}), 100 \mathrm{nM}$ thiamin, and $50 \mu \mathrm{g} / \mathrm{ml}$ kanamycin. After incubation at $37^{\circ} \mathrm{C}$ for $18 \mathrm{~h}$, cells were harvested by centrifugation, washed five times with thiamin-free MOPS medium, resuspended in $500 \mu \mathrm{l}$ of the same medium, serially diluted in 10-fold steps, and spotted on MOPS medium plates containing $0.2 \%$ glycerol, $0.02 \%$ arabinose, plus or minus $1 \mathrm{mM}$ Cys. Plates were then incubated at $37^{\circ} \mathrm{C}$ in aerobic and near-anerobic $\left(\mathrm{N}_{2}\right.$ containing $\left.\sim 1 \mathrm{ppm} \mathrm{O}_{2}\right)$ conditions as described [15]. Images were captured after $7 \mathrm{~d}$. For complementation assays with T. ammonificans THI4 in yeast, three independent clones of strain $\triangle T H I 4$

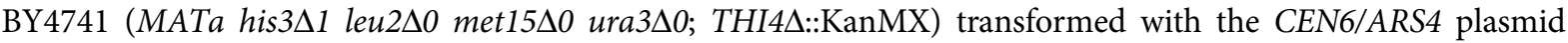
alone or containing T. ammonificans THI4 or yeast THI4 were used to inoculate $3 \mathrm{ml}$ of synthetic, complete medium (SC; yeast nitrogen base, USBiological cat. no. Y2036), drop-out mix (USBiological cat. no. D9540) minus histidine supplemented with $20 \mathrm{~g} / \mathrm{l}$ glucose, $5 \mathrm{~g} / \mathrm{l}$ ammonium sulfate, and $300 \mathrm{nM}$ thiamin. After $48 \mathrm{~h}$ of incubation at $30^{\circ} \mathrm{C}$ and $220 \mathrm{rpm}$, cells were pelleted $(3000 \mathrm{~g}, 5 \mathrm{~min})$, washed five times with thiamin-free SC minus histidine medium, resuspended in the same medium, and used to inoculate $3 \mathrm{ml}$ of thiamin-free SC minus histidine medium to an $\mathrm{OD}_{600 \mathrm{~nm}}$ of 0.05 . Growth was then monitored at $\mathrm{OD}_{600 \mathrm{~nm}}$.

\section{Purification and anaerobic reconstitution of T. ammonificans THI4}

T. ammonificans THI4 cloned in pBAD24 with an $\mathrm{N}$-terminal $\mathrm{His}_{6}$ tag was transformed into E. coli BL21 (DE3). A starter culture ( $15 \mathrm{ml} \mathrm{LB}$ plus $100 \mu \mathrm{g} / \mathrm{ml}$ ampicillin) was inoculated into $61 \mathrm{of} \mathrm{LB}$; THI4 expression was initiated at an $\mathrm{OD}_{600}$ of 0.6 with arabinose $(0.02 \% \mathrm{w} / \mathrm{v})$ and incubation was continued at $22^{\circ} \mathrm{C}$ for $20 \mathrm{~h}$. Cells were then harvested by centrifugation; pellets were resuspended in $50 \mathrm{ml}$ of lysis buffer $(20 \mathrm{mM}$ Tris$\mathrm{HCl}, \mathrm{pH} 8.0,500 \mathrm{mM} \mathrm{NaCl}, 2 \mathrm{mM}$ 2-mercaptoethanol) and lysed in a microfluidizer cell (14 $000 \mathrm{psi}, \mathrm{M}-110 \mathrm{~L}$ Pneumatic). The lysate was clarified by centrifugation $\left(18000 \mathrm{~g}, 30 \mathrm{~min}, 4^{\circ} \mathrm{C}\right)$ and applied to a $1-\mathrm{ml} \mathrm{Ni}-\mathrm{NTA}$ column (HisPur, ThermoFisher Scientific). After incubation at $4^{\circ} \mathrm{C}$ for $1 \mathrm{~h}$ the resin was washed with $50 \mathrm{ml}$ of lysis buffer and eluted with $6 \mathrm{ml}$ lysis buffer plus $250 \mathrm{mM}$ imidazole. The eluate was dialyzed against 11 of $20 \mathrm{mM}$ Tris- $\mathrm{HCl}, \mathrm{pH} 8.0,100 \mathrm{mM} \mathrm{NaCl}, 2 \mathrm{mM}$ 2-mercaptoethanol for $12 \mathrm{~h}$ and purified by anion exchange 
chromatography (HiTrap Q, GE Healthcare) with a linear gradient of $0-500 \mathrm{mM} \mathrm{NaCl}$ over $40 \mathrm{~min}$ and size-exclusion chromatography (HiLoad 16/600 Superdex 200, GE Healthcare) in $20 \mathrm{mM}$ Tris-HCl, pH 8.0, $100 \mathrm{mM} \mathrm{NaCl}, 2 \mathrm{mM} 2$-mercaptoethanol. Purified protein fractions were concentrated to $500 \mu \mathrm{l}(14 \mathrm{mg} / \mathrm{ml})$ and transferred to an anaerobic glovebox. Protein reconstitution and crystallization experiments were performed under pure argon and all solutions used were degassed and purged with argon before use. Purified enzyme ( $500 \mu \mathrm{l}, 14 \mathrm{mg} / \mathrm{ml}$ ) was incubated with 10 molar equivalents of ferrous ammonium sulfate at $22^{\circ} \mathrm{C}$ for $30 \mathrm{~min}$, followed by incubation with 10 molar equivalents each of $\mathrm{NAD}^{+}$and glycine for $1 \mathrm{~h}$.

\section{Crystallization and structure solution of T. ammonificans THI4}

Initial crystallization conditions were obtained using the hanging-drop method at $22^{\circ} \mathrm{C}$, with $2 \mu \mathrm{l}$ reconstituted enzyme and $2 \mu \mathrm{l}$ of $100 \mathrm{mM}$ HEPES-NaOH, pH 7.5, $200 \mathrm{mM} \mathrm{NaCl}$ and 35\% 2-methyl-2,4-pentanediol. Optimization of crystal morphology resulted in cubic shaped crystals in $\sim 2$ weeks with $100 \mathrm{mM}$ HEPES- $\mathrm{NaOH}, \mathrm{pH}$ 7.5, $200 \mathrm{mM} \mathrm{NaCl}, 35 \%$ 2-methyl-2,4-pentanediol, $10 \mathrm{mM}$ praseodymium acetate hydrate as the precipitant.

\section{Data collection, processing, and structure refinement}

Diffraction data were collected on beamline 23-ID-D of LS-CAT at Argonne National Laboratory Advanced Photon Source at a wavelength of $1.033 \AA$. Data were collected at $100^{\circ} \mathrm{K}$ and processed using XDS [24] to $2.3 \AA$ resolution in space group I121 (Supplementary Table S2). The structure was solved by molecular replacement using a homology model of Methanococcus igneus THI4 (PDB: $4 \mathrm{Y} 4 \mathrm{~N}, \sim 50 \%$ sequence identity). A model of $T$. ammonificans THI4 (four monomers per asymmetric unit) was iteratively built by combining AutoBuild (PHENIX [25]) with manual building in COOT [26]. Structure refinement was performed in PHENIX and REFMAC [25,27]. The parameter file for the bound glycine imine intermediate was generated using phenix. elbow [28]. The co-ordinates and structure factors are available from the Protein Data Bank (PDB ID: 7RK0).

\section{Results}

\section{Diversity of non-Cys THI4s and selection of representatives}

We first surveyed $\sim 1000$ prokaryotic THI4 proteins in the SEED [18] and UniRef90 [19] databases using BlastP and non-Cys THI4s [15] as query sequences. After removing entries that were truncated, redundant, or from unidentified organisms, there remained 199 unique sequences in which the active-site Cys position was occupied by His (171 cases) or by Met, Leu, Pro, Ala, Ser, Glu, Asp, Tyr, or Trp (28 cases) (Supplementary Table S3). The 199 sequences shared only $47 \%$ identity on average.

To analyze this sequence diversity and help select representatives to test for activity, we built SSNs using the Enzyme Function Initiative webtool $[20,29]$. The final SSN $\left(E\right.$-value $=10^{-5}$, alignment score $\left.=80\right)$ contained a series of clusters plus various singletons (Figure 2). Some clusters included non-Cys THI4s that have been tested for activity $[7,14,15]$ but others did not, indicating that much non-Cys THI4 sequence space remained to be sampled.

We selected 26 representative sequences from throughout the SSN and from organisms with different ecophysiologies (Figure 2 and Table 1). These sequences were about as diverse (45\% average identity) as the whole set of 199 and included THI4s described previously [7,14,15]. Fifteen of the selected sequences were bacterial and 11 were archaeal, eight were from organisms that require or tolerate $\mathrm{O}_{2}, 11$ were from mesophiles, 15 were from thermophiles, 22 were from habitats known or likely to be sulfide-rich, and four were from habitats likely to be sulfide-poor (Table 1).

\section{Genomic context of non-Cys THI4 genes}

The selected THI4s all came from genomes that encode ThiE or ThiN (Supplementary Figure S1), indicating capacity to synthesize thiamin from its thiazole and pyrimidine precursors. All genomes also had ThiD and all but one had ThiC (Supplementary Figure S1), respectively, indicating capacity to utilize or produce the pyrimidine precursor. Nearly all the selected THI4s, therefore, came from organisms with complete thiamin synthesis pathways. Only six genomes encoded the alternative thiazole synthase ThiG, so that THI4 was typically the only identifiable endogenous source of the thiazole precursor. Three of the selected archaeal THI4 genes abut a gene coding for a protein from the TRASH family, whose members bind nonferrous heavy metals, with characterized examples involved in zinc, copper, cadmium, and/or mercury transport/resistance [30-35] 


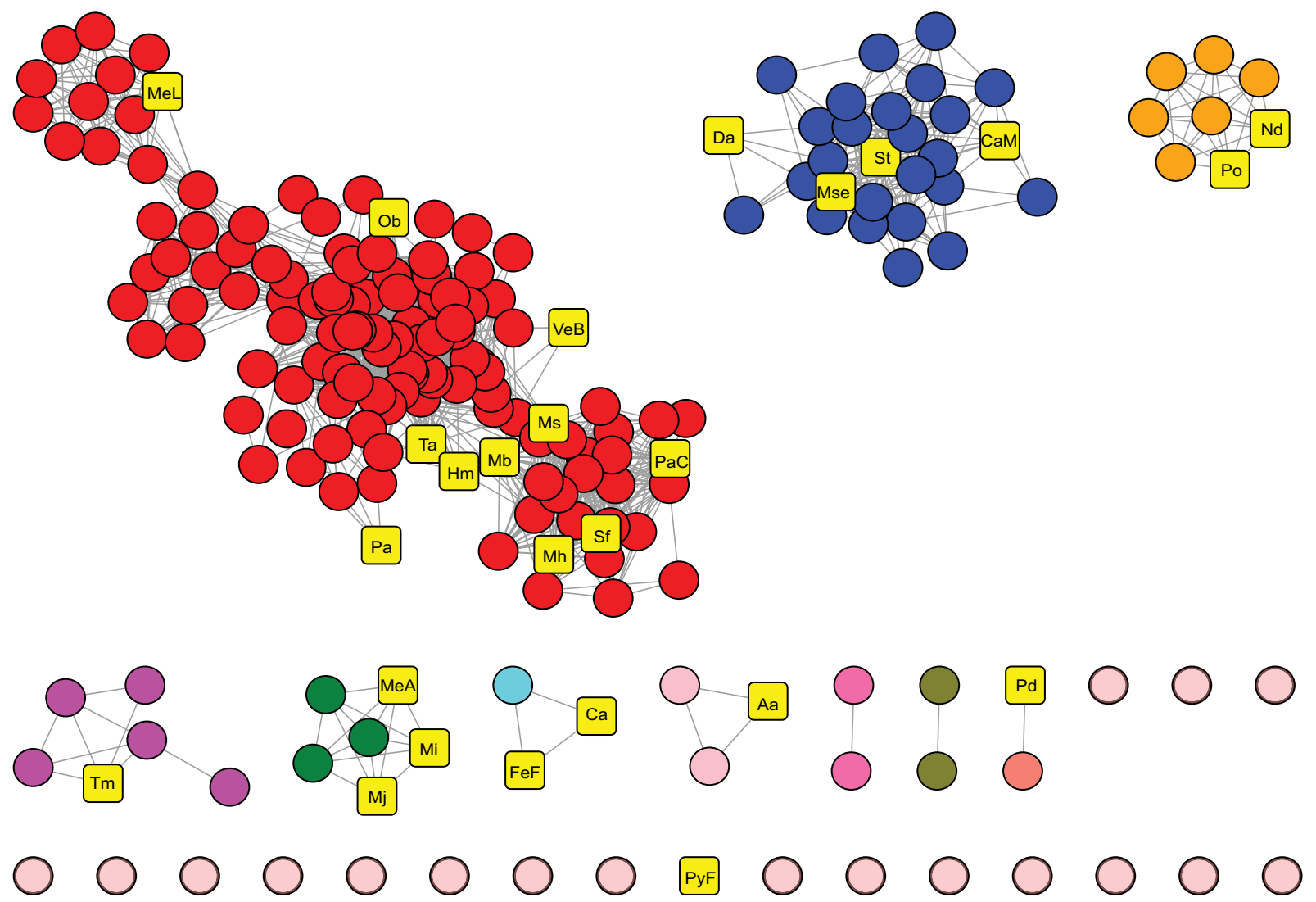

Figure 2. Sequence similarity network (SSN) of 199 diverse non-Cys THI4s.

Each node in the SSN corresponds to a single sequence; each edge (gray lines) represents a pair-wise connection between two sequences at a BLAST $E$-value $<1 \times 10^{-5}$. Lengths of edges are not significant, except that tightly clustered groups share more similarity than sequences with only a few connections. The 26 representative sequences selected for testing are shown as yellow squares; organism name abbreviations are as in Table 1.

(Supplementary Figure S1). Similar clustering occurs in many other archaea and bacteria (Figure 3A). The TRASH proteins in these clusters have two adjacent Cys residues in addition to the four canonical metal-binding Cys residues in the TRASH motif (Cys- $\mathrm{Xaa}_{2}-\mathrm{Cys}-\mathrm{Xaa}_{19-22}-\mathrm{Cys}-\mathrm{Xaa}_{3}-\mathrm{Cys}$ ) [30] (Figure 3B); these extra Cys could help ligand a six-co-ordinate metal such as cobalt, nickel, or iron. Furthermore, non-Cys THI4 and TRASH genes cluster with genes for proteins from families that transport or metabolize cobalt or nickel [36-39] (Figure 3A). Non-Cys THI4s are thus more strongly genomically associated with nonferrous metals than with iron.

\section{Functional complementation tests of THI4 activity}

The 26 selected THI4s were recoded for E. coli and inserted into pBAD24 [23]; the resulting constructs were then introduced into an E. coli $\Delta$ thiG strain [15]. To check THI4 expression, cells were grown in thiaminsupplemented minimal medium and harvested for gel analysis of the soluble and insoluble fractions (Figure 4A,B and Supplementary Figure S2). Of the 26 THI 4 s, 23 expressed well, with $\geq 85 \%$ in soluble form (Table 1) and were advanced to testing for thiazole synthase activity by complementation.

Complementation tests were run in air or in $\mathrm{N}_{2}$ containing $\sim 1 \mathrm{ppm} \mathrm{O}_{2}$, plus or minus supplementation with $1 \mathrm{mM}$ Cys to increase intracellular sulfide level [40]. Sixteen strains showed clear growth in air, particularly when supplemented with Cys while seven did not, and no strain showed clear growth in $\sim 1 \mathrm{ppm} \mathrm{O}_{2}$ but not in air (Figure 4C and Supplementary Figure S3). The enhanced growth with Cys supplementation fits with use of sulfide as sulfur donor [5,15]. The complementation tests in air thus split the THI4s into one group with readily detectable activity (henceforth: aerotolerant THI4s) and another with little or none. Both groups 
Table 1. Ecophysiology of the prokaryote sources of the THI4s selected for testing

\begin{tabular}{|c|c|c|c|c|}
\hline Organism (abbreviation) & Natural habitat ${ }^{1}$ & $\mathrm{O}_{2}$ adaptation & Temperature & Sulfide $^{1,2}$ \\
\hline \multicolumn{5}{|l|}{ THI4s with readily detectable activity } \\
\hline \multicolumn{5}{|l|}{ Bacteria } \\
\hline Fervidicola ferrireducens (FeF) & Thermal aquifer & Obligate anaerobe & Thermophile & Low \\
\hline Thermovibrio ammonificans (Ta) & Hydrothermal vent & Anaerobe & Thermophile & High \\
\hline Hippea maritima $(\mathrm{Hm})$ & Hydrothermal vent & Anaerobe & Thermophile & High \\
\hline Thermotoga maritima (Tm) & Hot marine sediment & Anaerobe & Thermophile & High \\
\hline Caldanaerovirga acetigignens (Ca) & Hot springs & Anaerobe & Thermophile & High \\
\hline Mucinivorans hirudinis (Mh) & Leech gut & Anaerobe & Mesophile & High \\
\hline Parabacteroides chinchillae (PaC) & Chinchilla gut & Anaerobe & Mesophile & High \\
\hline Verrucomicrobia bacterium (VeB) & Soil & Anaerobe & Mesophile & Low \\
\hline Pseudoramibacter alactolyticus (Pa) & Abscesses & Anaerobe & Mesophile & High \\
\hline Marinilabilia salmonicolor (Ms) & Marine mud & Facultative aerobe & Mesophile & High \\
\hline Saccharicrinis fermentans (Sf) & Marine mud & Facultative aerobe & Mesophile & High \\
\hline Candidatus Marinimicrobia (Mb) & Sea water column & Facultative aerobe & Mesophile & Low \\
\hline \multicolumn{5}{|l|}{ Archaea } \\
\hline Pyrodictium delaneyi $(\mathrm{Pd})$ & Hydrothermal vent & Obligate anaerobe & Thermophile & High \\
\hline Methanococcus jannaschii (Mj) & Hydrothermal vent & Obligate anaerobe & Thermophile & High \\
\hline Methanococcus aeolicus (MeA) & Marine sediments & Obligate anaerobe & Mesophile & High \\
\hline Methanococcus igneus (Mi) & Hydrothermal vent & Anaerobe & Thermophile & High \\
\hline \multicolumn{5}{|l|}{ THI4s with little to no detectable activity } \\
\hline \multicolumn{5}{|l|}{ Bacteria } \\
\hline Candidatus Omnitrophica (Ob) & Hydrothermal vent & Anaerobe & Thermophile & High \\
\hline Poribacteria sp. WGA-A3 (Po) & Sponge symbiont & Facultative aerobe & Mesophile & Low \\
\hline Nitrospira defluvii (Nd) & Sewage sludge & Aerobe & Mesophile & High \\
\hline \multicolumn{5}{|l|}{ Archaea } \\
\hline Desulfurococcus amylolyticus (Da) & Hot springs & Obligate anaerobe & Thermophile & High \\
\hline Candidatus Aenigmarchaeota (Aa) & Hot springs & Anaerobe & Thermophile & High \\
\hline Pyrolobus fumarii (PyF) & Hydrothermal vent & Facultative microaerobe & Thermophile & High \\
\hline Metallosphaera sedula (Mse) & Acidic hot springs & Aerobe & Thermophile & High \\
\hline \multicolumn{5}{|l|}{ Poorly expressed archaeal THI4S ${ }^{\mathrm{C}}$} \\
\hline Sulfurisphaera tokodaii (St) & Hot springs & Obligate aerobe & Thermophile & High \\
\hline Methanofollis liminatans (MeL) & Wastewater bioreactor & Obligate anaerobe & Mesophile & High \\
\hline Caldivirga maquilingensis (CaM) & Acidic hot spring & Facultative microaerobe & Thermophile & High \\
\hline
\end{tabular}

included THI4s from mesophiles and thermophiles, aerobes and anaerobes, and organisms from high- and low-sulfide habitats (Table 1). There was hence no evident correlation between THI4 aerotolerance and source organism ecophysiology. Neither was aerotolerance correlated with the residue (His, Met, Leu, Tyr, or Ser) that replaces Cys in the active site or with the number of Cys and Met residues, which can affect oxidative instability [41,42] (Supplementary Table S3). To summarize: the complementation data establish that some catalytic THI4s operate quite well (although not optimally, see below) in aerobic conditions but do not suggest why others do not. One of many possible causes is that the redox environment in E. coli differs enough from that in 


\section{A} Metallosphaera
sedula
(Crenarcheota)
Geoglobus
ahangari 234
(Euryarchaeota)

Methanopyrus

kandleri

(Euryarchaeota)
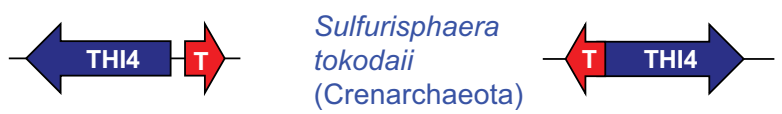

Pyrobaculum arsenaticum

(Crenarchaeota)
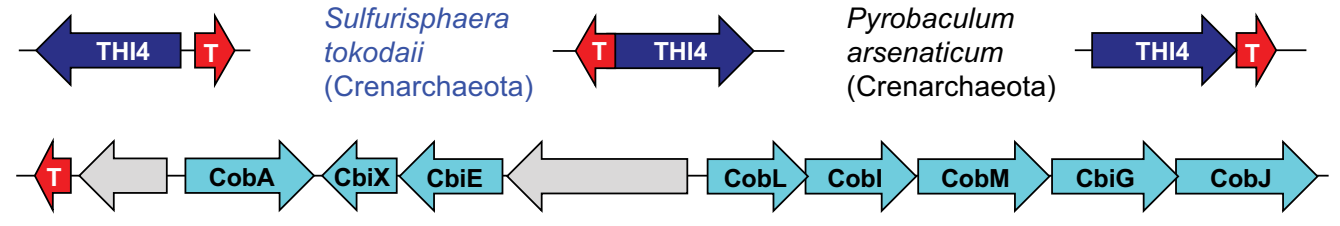

Staphylothermus marinus

(Crenarchaeota)
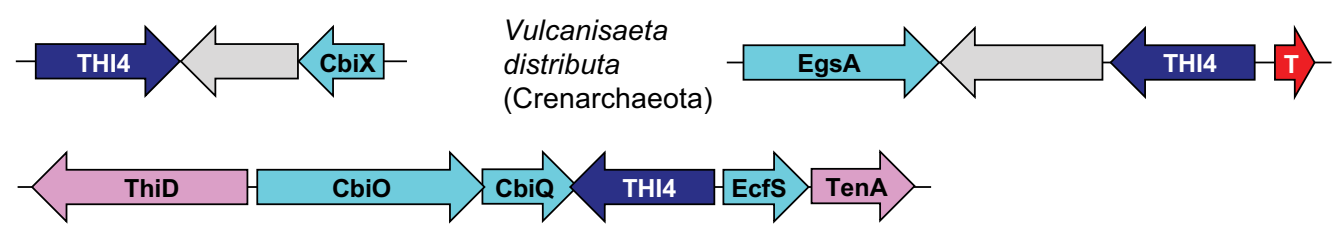

Vulcanisaeta $s p$.

OSP_8

(Crenarcheota)

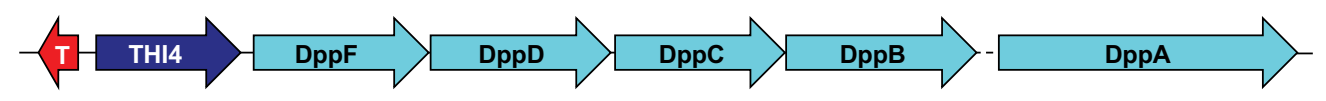

Pyrodictium delaneyi Su06

(Crenarcheota)

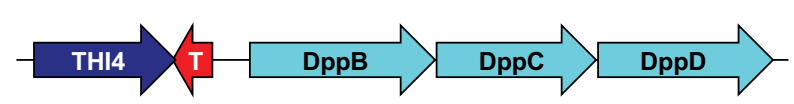

Caldimicrobium

thiodismutans

(Thermodesulfobacteria)

Pyrobaculum oguniense (Crenarchaeota)

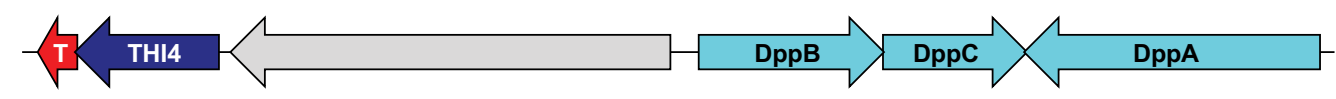

Nitrososphaerales archaeon SpSt-912
(Thaumarchaeota)

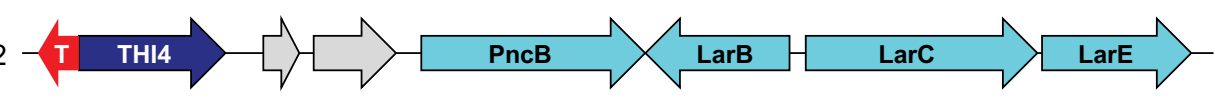

\section{B}

M. sedula

S. tokodaii

P. arsenaticum

P. oguniense

V. sp. OSP_8

V. distribüta

P. delaneyi

G. ahangari

N. Spst-912

C. thiodismutans

Figure 3. Association of THI4 genes with TRASH genes and cobalt- or nickel-related genes.

(A) Chromosomal gene clustering arrangements. Names of organisms whose THI4s were tested are in blue. T, TRASH protein gene. Genes colored aqua encode homologs of nickel- and/or cobalt-related proteins: CobA, uroporphyrin-III C-methyltransferase, involved in cobalamin and sirohaem synthesis; CbiX, sirohydrochlorin nickel/cobalt chelatase, which inserts nickel or cobalt into tetrapyrroles; CbiE, involved in cobalamin synthesis; a cobalamin synthesis operon encoding CobL, Cobl, CobM, CbiG, CobJ, plus (not shown) CbiC and CbiD; CbiO, CbiQ, and EcfS, the A+ A', T, and S components of a cobalt energy coupling-factor (ECF) transporter. DppA-D,F, subunits of $A B C$ transporters for oligopeptides, nickel, or (rarely) cobalt; LarBCE, nickel-pincer nucleotide (NPN) cofactor synthesis genes; PncB, synthesis enzyme for the NPN precursor NaAD; EgsA,

glycerol-1-phosphate dehydrogenase, which has a nickel or zinc cofactor; gray genes have no known nickel or cobalt associations. Genes colored lilac encode thiamin synthesis or salvage enzymes. (B) Alignment of the TRASH proteins encoded by genes in part A. The Cys residues of the extended TRASH motif (Cys-Xaa 2 -Cys-Xaa ${ }_{19-22}-$ Cys-Xaa 3 -Cys) are in yellow; the two extra Cys residues are in red.

the THI4 source, especially for extremophiles (Table 1) [43-45], to disrupt protein disulfide formation or metal insertion and coordination [45-47] and thus prevent THI4 expression in its native form.

It is important to note that supplementation with thiamin markedly stimulated the growth of all strains (compare NA and + Thi columns in air in Figure 4C and Supplementary Figure S3). This stimulation shows that the in vivo activity of THI4s with complementing activity did not fully meet the demand for ADT despite 


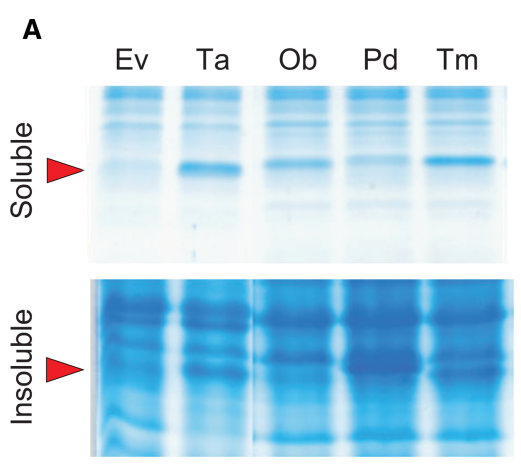

C

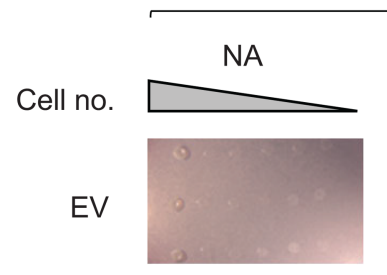

TaTHI4

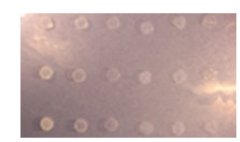

TmTHI4

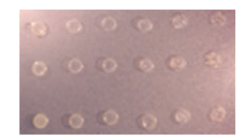

PdTHI4

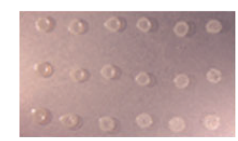

ObTHI4

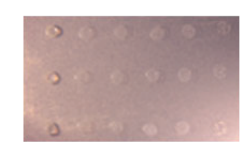

Air
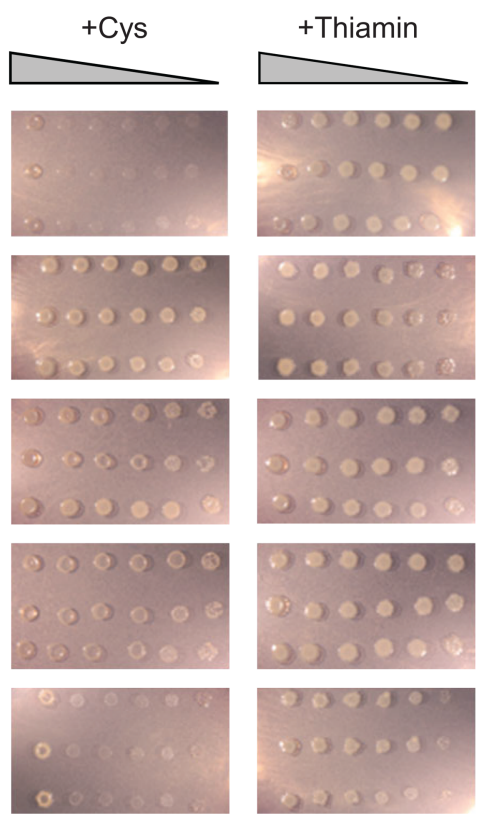

B

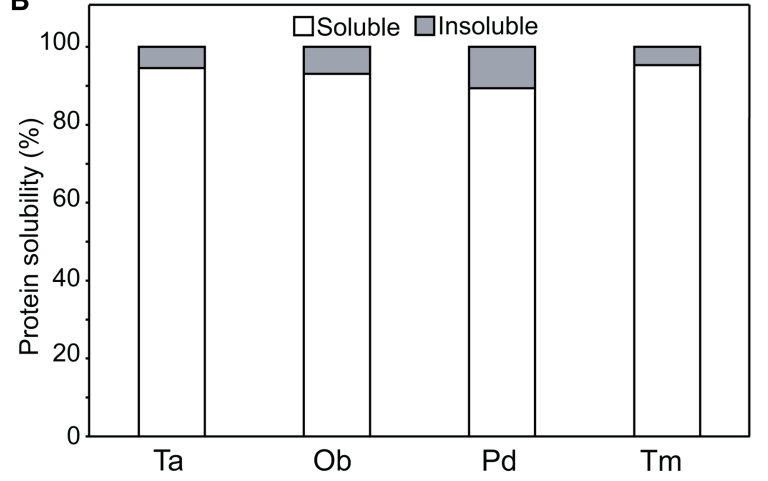

$\sim 1 \mathrm{ppm} \mathrm{O}_{2}$

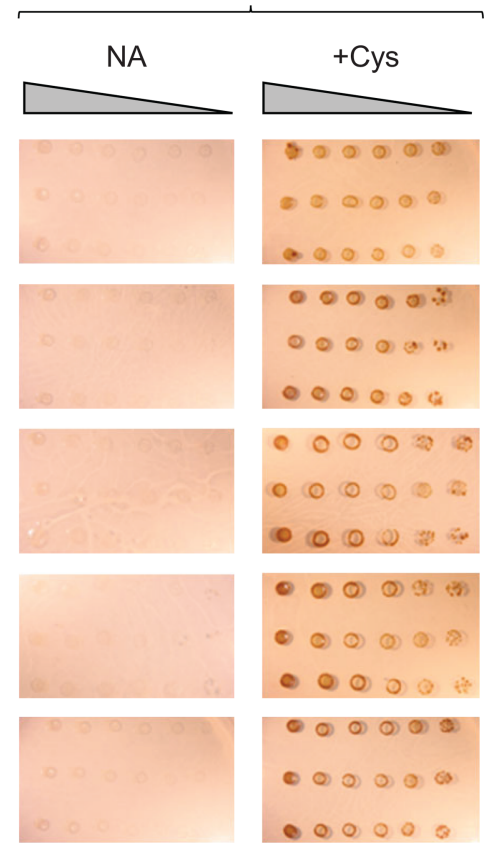

Figure 4. Soluble expression and functional complementation tests of non-Cys THI4s.

(A) Gel analysis of soluble and insoluble expression in E. coli of representative THI4s. Soluble and insoluble fractions of cells were run on $15 \%$ gels, stained with Coomassie blue, and scanned to quantify the THI4 band, for which purified Thermovibrio ammonificans (Ta) THI4 served as a marker (arrow). Organism abbreviations are as in Table 1. (B) Quantification of the solubility (\%) of the THI4s from (A). (C) Tests of functional complementation of an E. coli $\Delta$ thiG strain by the THI4s from (A) or the empty vector (EV). TaTHI4, TmTHI4, and PdTHI4 had clearly detectable activity in air while ObTHI4 did not; none had more than slight activity in $\sim 1 \mathrm{ppm} \mathrm{O}_{2}$. Overnight cultures of three independent clones per construct were 10-fold serially diluted and spotted on plates of MOPS minimal medium containing $0.2 \%(\mathrm{w} / \mathrm{v})$ glycerol and $0.02 \%(\mathrm{w} / \mathrm{v})$ arabinose with no additions (NA) or plus $1 \mathrm{mM}$ Cys or $100 \mathrm{nM}$ thiamin. The medium used for culture in $\sim 1 \mathrm{ppm} \mathrm{O}_{2}$ contained $40 \mathrm{mM}$ nitrate as electron acceptor. Cells were cultured in air or under $\mathrm{N}_{2}$ containing $\sim 1 \mathrm{ppm} \mathrm{O}_{2}$. Images were captured after incubation at $37^{\circ} \mathrm{C}$ for $7 \mathrm{~d}$. The high background in the $\sim 1 \mathrm{ppm}_{2}+$ Cys treatment is due to staining of the inoculum cells.

their high expression levels (Figure 4A and Supplementary Figure S2), i.e. that there is room for activity improvement, at least when no Cys is supplied. We revisit this point later.

\section{Protein structure of the aerotolerant T. ammonificans THI4}

As structural data for THI4s with little or no complementing activity in air would be hard to interpret in terms of aerotolerance alone since failure has other possible causes, we explored molecular features associated with 
aerotolerance by determining the crystal structure of the complementation-active THI4 from T. ammonificans (TaTHI4) (PDB: 7RK0). This bacterium is an anaerobe but is likely to be intermittently exposed to $\mathrm{O}_{2}$ in its habitat near the oxic/anoxic interface in hydrothermal vents, and encodes enzymes to detoxify reactive oxygen species (ROS), including catalase/peroxidase, cytochrome $c$ peroxidase, and cytochrome $b d$ complex $[48,49]$. This makes the TaTHI4 structure a potentially informative 'routinely $\mathrm{O}_{2}$-exposed' comparison with the structures of 'never $\mathrm{O}_{2}$-exposed' THI4s from the archaeal methanogens $M$. igneus, M. jannaschii, and Methanothermococcus thermolithotrophicus [7,50]. Like T. ammonificans, these organisms are anaerobic thermophiles from hydrothermal vents [48,51] but, unlike T. ammonificans, they inhabit the anoxic region of the vent plume and lack the ROS defense genes present in T. ammonificans as well as heme- and manganesecatalase genes (Supplementary Table S4). Furthermore, M. igneus and M. jannaschii THI4s had similar complementing activity in E. coli to TaTHI4 (Supplementary Figure S3), meaning that differences among their recombinant protein structures are unlikely to be artifacts of misfolding in the heterologous host. In comparing the TaTHI4 structure with those of the three archaeal methanogens we made the reasonable assumption that these non-Cys THI4s are all catalytic, as M. jannaschii THI4 has been shown to be [14].

Protein crystals of TaTHI4 diffracted to $2.3 \AA$ resolution in space group I121 and the structure was solved by molecular replacement (Supplementary Table S2) using M. igneus THI4 as a search model (PDB: 4Y4N) [7]. In common with the methanogen THI4s, TaTHI4 is an overall homooctamer with four monomers per asymmetric unit (Figure 5A). The octamer is tightly packed as a two-layer ring structure with approximate dimensions $73 \times 86 \AA$ (height $\times$ width) enclosing a $\sim 26 \AA$ diameter pore. The monomer structure (Figure $5 \mathrm{~B}$ ) consists of a long $\alpha$-helix (residues 6-24) and a barrel-like core domain sandwiched by helix bundles (Figure $5 \mathrm{C}$ ). The barrel-like core comprises a central five-stranded $\beta$-sheet with $\beta 6, \beta 2, \beta 1, \beta 12, \beta 13$ topology, flanked by a twisted antiparallel $\beta$-sheet $(\beta 7, \beta 10, \beta 11)$ connecting $\alpha 6$ and a four-helix bundle $(\alpha 9, \alpha 12, \alpha 2, \alpha 5)$. The barrel core is capped by a variation of the Rossmann fold $\beta$-hairpin motif. The C-terminal topology is similar to the canonical Rossman fold with an inversion of strands. TaTHI4 is thus structurally homologous to the previously solved methanogen THI4s [7,50].

The enzyme active site lies in a channel formed by two helix bundles and is capped by a loop from an adjoining monomer. Clear density for the TaTHI4 treated with NAD and glycine shows the expected glycine imine intermediate [7] in the active site (Figure 6A). This intermediate adopts an elongated conformation with the adenine moiety positioned in the barrel-like core domain. Binding interactions between the intermediate and TaTHI4 include a hydrogen bond between N6 of adenine and the side chain of Ser178, a bidentate hydrogen bond with the $2^{\prime}$ and $3^{\prime}$ hydroxyls and Glu56, a second hydrogen bond between Arg58 and the $2^{\prime}$ hydroxyl, along with a stabilizing interaction of the glycine imine carboxylate and Arg239 (Figure 6A). Based on our reconstitution conditions, the bound metal in the active site can be assigned as ferrous iron, Fe (II). The iron is co-ordinated in a pseudooctahedral geometry with three sites occupied by the pincer-type ligand of the bound glycine imine, axial ligands of His175 and Asp160, and a predicted water molecule (Figure 6B,C).

To probe the role of the Met158 residue that in TaTHI4 occupies the position of the sacrificial Cys, we changed Met158 to His, Lys, or Cys and assayed complementing activity (Supplementary Figure S4). The mutant enzymes performed similarly to wild-type TaTHI4 in both air and $\sim 1 \mathrm{ppm} \mathrm{O}_{2}$ atmospheres, with or without Cys supplementation. This result confirms the inference from natural variation in this residue (Supplementary Table S3) that it has no role in catalysis. That the Cys form did not have greater activity in the absence of Cys supplementation suggests that it cannot operate efficiently in suicide mode and still depends on sulfide as sulfur source, as was the case for M. jannaschii THI4 [7].

\section{Investigation of TaTHI4 metal preference in vivo in E. coli and yeast}

As we could not obtain quantifiable in vitro activity [14] from reconstituted TaTHI4, we sought to test metal cofactor preference in vivo. Adding $100 \mu \mathrm{M}$ cobalt, nickel, zinc, or manganese to the medium of E. coli expressing TaTHI4 (or five other THI4s) did not detectably affect complementing activity in air (Supplementary Figure S5). This result does not indicate which metal is preferred, but does show that the supply of this metal is not limiting in $E$. coli grown with normal trace metal supplementation.

We also tested metal preference using yeast, which resembles $E$. coli in having native cobalt-dependent enzymes and cobalt uptake systems [52] but differs in having no native nickel enzymes or high-affinity nickel uptake system and in needing an added nickel transporter to express a foreign nickel enzyme in active form [53]. We, therefore, tested TaTHI4 for the ability to complement a yeast $\Delta$ THI4 strain. The observed 

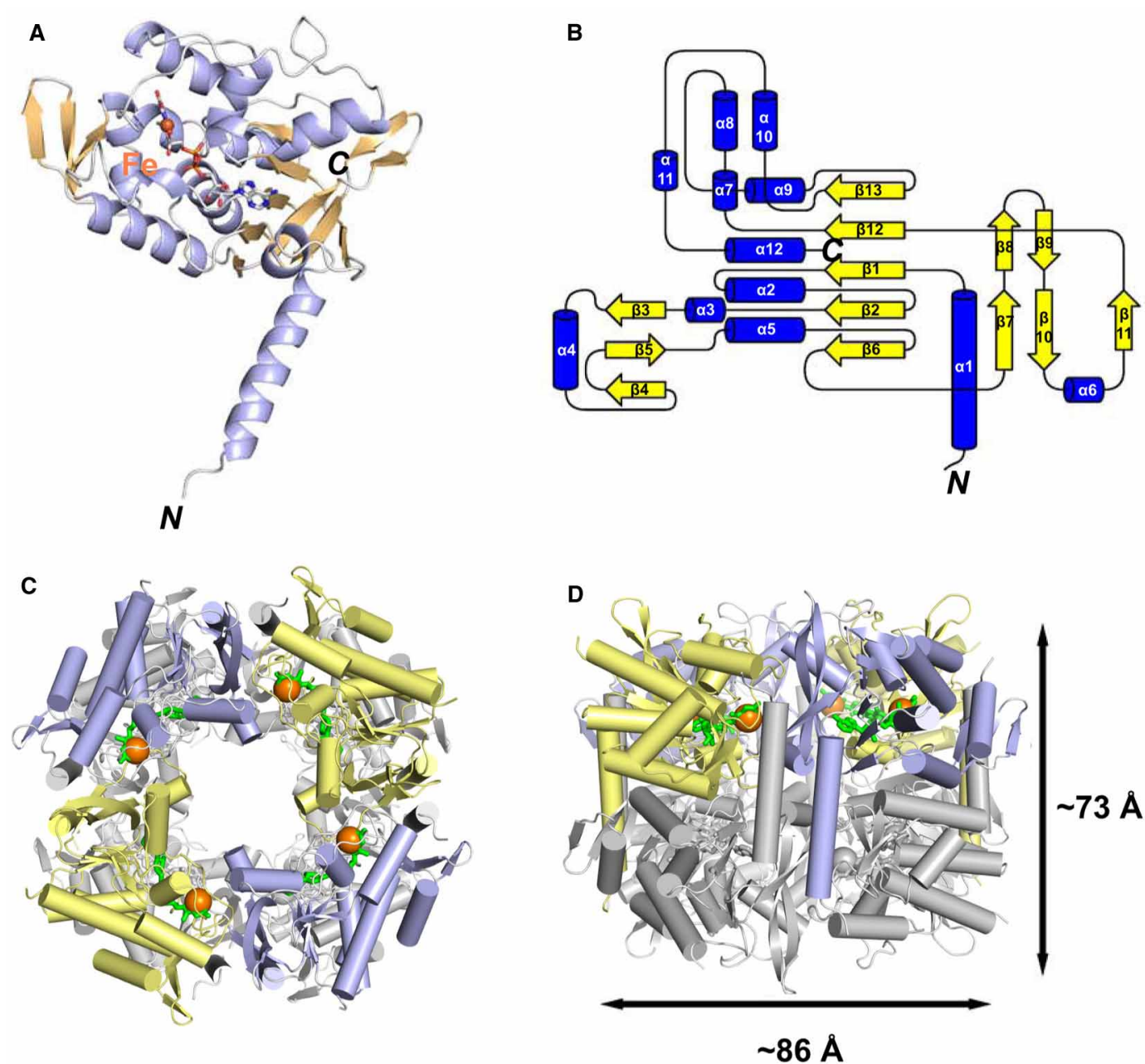

Figure 5. Structure of the aerotolerant non-Cys THI4 from Thermovibrio ammonificans.

(A) Ribbon diagram of the TaTHI4 monomer with bound glycine imine shown as sticks; $\alpha$-helices are colored blue, $\beta$-strands are colored beige; $\mathrm{Fe}(\mathrm{II})$ is shown as a brown sphere. (B) Topology diagram of TaTHI4 with $\alpha$-helices in blue and $\beta$-strands in yellow. (C,D) The biologically relevant TaTHI4 homooctamer shown in ribbon representation in alternate top and side views with the metal $\mathrm{Fe}(\mathrm{II})$ indicated as orange spheres and the glycine imine as green sticks. One tetramer is blue/yellow and the other is colored gray.

complementation (Supplementary Figure S6) proves that TaThi4 has access to its metal cofactor and implies that this metal is more likely to be cobalt than nickel if it is not iron.

\section{Discussion}

The biochemically characterized catalytic THI4s come from archaea of anoxic, sulfide-rich, reducing environments, use an $\mathrm{O}_{2}$-sensitive sulfur donor (sulfide) and cofactor (ferrous iron), and are thermophilic [7,14]. It was consequently a priori doubtful whether catalytic prokaryotic THI4s could function in mild, fully aerobic conditions, and recent evidence [5] that putatively catalytic plant THI4s are expressed only at severely hypoxic stages of seed development reinforced this doubt.

However, our survey of THI4 sequences and their provenances began to dispel the doubt because non-Cys THI4s confidently predicted from genomic context to be functional thiazole synthases were found in aerobic or aerotolerant organisms from mild environments. Confirmation that certain non-Cys THI4s - the majority of those tested, in fact - can function in air came from complementation experiments, which also indicated that these THI4s use sulfide, or a sulfide metabolite, as sulfur donor. 

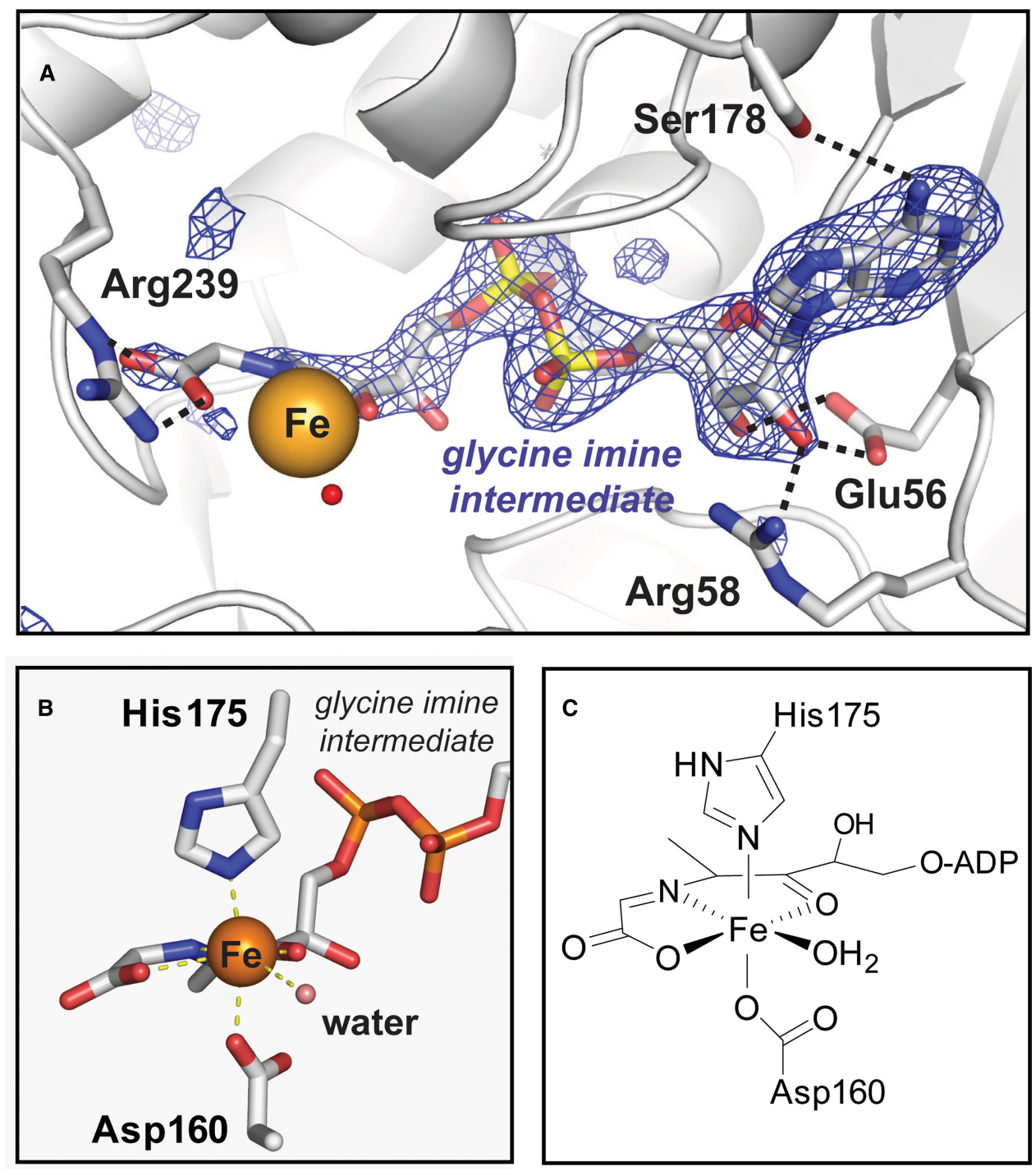

Figure 6. The active site of Thermovibrio ammonificans THI4.

(A) Interactions of the bound glycine intermediate with the TaThi4 protein active site. A glycine imine intermediate omit map, Fo-Fc contoured at $3.5 \sigma$, is shown in blue. (B) Ligand geometry around the metal center with a near-octahedral coordination environment as diagrammed in (C).

Having established that certain non-Cys THI4s can - unexpectedly - operate in mild, fully aerobic conditions, we investigated characteristics that enable them to do so. We could dismiss any role, positive or negative, for thermophily because complementing activity varied similarly in thermophiles and mesophiles (Table 1). There was likewise no association between complementing activity and the $\mathrm{O}_{2}$ adaptation of the source organism (Table 1). Nor did the residue that replaces the sacrificial Cys or the number of Cys or Met residues appear to be important (Supplementary Table S3).

What then, might confer aerotolerance? The comparative genomic analysis provided a clue by associating non-Cys THI4s with proteins that bind, transport, or metabolize nonferrous transition metals, notably cobalt or nickel (Figure 3A), and the observed complementing activity of TaTHI4 in yeast favored the possibility that the metal cofactor is cobalt (Supplementary Figure S6). Neither the comparative genomics nor the yeast complementation data ruled out a ferrous iron cofactor, however.

Comparing the TaTHI4 structure - the first deposited for a bacterial THI 4 - with those of the ecologically less $\mathrm{O}_{2}$-exposed THI4s from archaeal methanogens (PDBs: 4Y4M, 4Y4N, 6HK1) [7,50] did not provide strong evidence on the aerotolerance phenomenon. The active sites are largely identical, with one major difference, 
the variation of residues (Met vs. His) replacing the Cys residue of suicide THI4s. Variants at this position, however, do not correlate with aerotolerance (Supplementary Table S3). The octamer structures show similar dimensions of the overall barrel-like architecture and similar surface electrostatic charge. Notable differences are two extended loop regions in TaTHI4 at the entrance to the large active-site pore (residues 134-142 and 186-197); however, based on the pore diameter, these changes are unlikely to affect $\mathrm{O}_{2}$ diffusion. An analogous comparision of the structures of $\mathrm{O}_{2}$-sensitive and $\mathrm{O}_{2}$-tolerant homologs of [NiFe] hydrogenase showed distinct, well-defined tunnels to the metal center [54,55]. In this case, structural variations of the tunnel were predicted to regulate $\mathrm{O}_{2}$ diffusion to the reactive metal center. All THI4s have a large pore ( 26 $)$ linking the active site to solvent, precluding variation in $\mathrm{O}_{2}$ diffusion. The lack of dissimilar structural features favors the alternative possibility of a role for the active site metal in $\mathrm{O}_{2}$ tolerance.

Semi-quantitative in vitro assay of M. jannaschii THI4 activity [14] showed that cobalt supported $\sim 60 \%$, and nickel $\sim 25 \%$, of the activity given by iron. The presented TaTHI4 structure contains an obligate bound $\mathrm{Fe}(\mathrm{II})$ atom, based on $\mathrm{Fe}$ (II) being the only metal present during protein reconstitution. The ligand geometry in the structure (Figure 6) does not preclude other metals, including $\mathrm{Co}(\mathrm{II})$ or $\mathrm{Ni}(\mathrm{II})$. A bound $\mathrm{Ni}(\mathrm{II})$ would be predicted to favor a square pyrimidal geometry [56] over the octahedral although the active site could possibly accommodate a minor shift in ligand position to square pyrimidal. There is solid precedent for octahedral Co (II), including colbalamin enzymes and methylmalonyl-CoA carboxytransferase [57]. In terms of THI4 chemistry, $\mathrm{Co}(\mathrm{II})$ is less likely to react deleteriously with molecular $\mathrm{O}_{2}$ than $\mathrm{Ni}(\mathrm{II})$ and $\mathrm{Fe}(\mathrm{II})$. Contrasting with many examples in which mononuclear $\mathrm{Fe}(\mathrm{II})$ reacts with $\mathrm{O}_{2}$ to generate reactive $\mathrm{O}$-atom transfer species in metalloenzymes and model compounds [58,59], reaction of $\mathrm{Co}(\mathrm{II})$ with $\mathrm{O}_{2}$ has strong precedent for reversible formation of $\mathrm{Co}(\mathrm{III})$-superoxide species in the absence of additional reducing equivalents or protonation [60], implying that replacing iron with cobalt could provide a mechanism for the observed $\mathrm{O}_{2}$ tolerance. In addition, a $\mathrm{Ni}$ (II)-SH intermediate in the catalytic cycle would be particularly stable [61], disfavoring catalysis. Other metals are known to be able to perform THI4 chemistry, notably Zn(II), but the ligand geometry and metal preference analysis of $M$. jannaschii THI4 [14] argue against a tetrahedral liganded metal.

In summary, the comparative genomics, functional complementation, and structural evidence collectively implicate the bound metal as a natural determinant of THI4 aerotolerance, with $\mathrm{Co}$ (II) the best candidate although $\mathrm{Ni}$ (II) cannot be rigorously excluded. Definitive evidence on this point will require the development of quantitative in vitro assays for THI4 activity. Besides the nature of the metal inserted, there may well be other determinants of aerotolerance. We hope to identify such determinants, and to gain insight into the metal cofactor, from ongoing continuous directed evolution experiments [62] to improve the complementing activity of native non-Cys THI4s. If - as seems likely [63] — such improvement is possible, there is a realistic prospect of replacing suicidal plant THI4s with catalytic THI4s that work well in aerobic conditions and thus slash the energy cost of thiamin synthesis.

\section{Data Availability}

Co-ordinates and structure factors of the T. ammonificans THI4 crystal structure have been deposited in PDB under code 7RKO.

\section{Competing Interests}

The authors declare that there are no competing interests associated with the manuscript.

\section{Funding}

This work was supported primarily by the U.S. Department of Energy, Office of Science, Basic Energy Sciences, under Award DE-SC0020153, and by an endowment from the C.V. Griffin Sr. Foundation.

\section{Open Access}

Open access for this article was enabled by the participation of University of Florida in an all-inclusive Read \& Publish pilot with Portland Press and the Biochemical Society.

\section{Author Contribution}

A.D.H., J.J., and S.D.B. designed the research; J.J., J.-D.G.-G., and B.J.L. performed assays; J.J. and A.D.H. ran bioinformatic analyses; Q.L., Y.H., and S.D.B. carried out structural analysis; A.D.H. wrote the article with input from J.J. and S.D.B. 


\section{CRediT Author Contribution}

Andrew D. Hanson: Conceptualization, formal analysis, supervision, funding acquisition, investigation, writing - original draft. Jaya Joshi: Conceptualization, formal analysis, investigation, writing - review and editing. Qiang Li: Formal analysis, investigation, writing - review and editing. Jorge D. García-García: Investigation, writing - review and editing. Bryan J. Leong: Investigation, writing - review and editing. You Hu: Investigation, writing - review and editing. Steven D. Bruner: Conceptualization, formal analysis, supervision, investigation, writing - original draft, writing - review and editing.

\section{Acknowledgements}

We thank Prof. Leslie J. Murray for helpful discussions.

\section{Abbreviations}

ADT, adenylated carboxythiazole; IPTG, Isopropyl $\beta$-D-1-thiogalactopyranoside; ROS, reactive oxygen species; SSN, sequence similarity network.

\section{References}

1 Begley, T.P., Ealick, S.E. and McLafferty, F.W. (2012) Thiamin biosynthesis: still yielding fascinating biological chemistry. Biochem. Soc. Trans. 40, 555-560 https://doi.org/10.1042/BST20120084

2 Hanson, A.D., Amthor, J.S., Sun, J., Niehaus, T.D., Gregory, III, J.F., Bruner, S.D. et al. (2018) Redesigning thiamin synthesis: prospects and potential payoffs. Plant Sci. 273, 92-99 https://doi.org/10.1016/j.plantsci.2018.01.019

3 Chatterjee, A., Abeydeera, N.D., Bale, S., Pai, P.J., Dorrestein, P.C., Russell, D.H. et al. (2011) Saccharomyces cerevisiae THI4p is a suicide thiamine thiazole synthase. Nature 478, 542-546 https://doi.org/10.1038/nature10503

4 Faou, P. and Tropschug, M. (2004) Neurospora crassa CyPBP37: a cytosolic stress protein that is able to replace yeast Thi4p function in the synthesis of vitamin B1. J. Mol. Biol. 344, 1147-1157 https://doi.org/10.1016/j.jmb.2004.09.097

5 Joshi, J., Beaudoin, G.A.W., Patterson, J.A., García-García, J.D., Belisle, C.E., Chang, L.Y. et al. (2020) Bioinformatic and experimental evidence for suicidal and catalytic plant THI4s. Biochem. J. 477, 2055-2069 https://doi.org/10.1042/BCJ20200297

6 Hwang, S., Cordova, B., Chavarria, N., Elbanna, D., McHugh, S., Rojas, J. et al. (2014) Conserved active site cysteine residue of archaeal THI4 homolog is essential for thiamine biosynthesis in Haloferax volcanii. BMC Microbiol. 14, 260 https://doi.org/10.1186/s12866-014-0260-0

7 Zhang, X., Eser, B.E., Chanani, P.K., Begley, T.P. and Ealick, S.E. (2016) Structural basis for iron-mediated sulfur transfer in archael and yeast thiazole synthases. Biochemistry 55, 1826-1838 https://doi.org/10.1021/acs.biochem.6b00030

8 Godoi, P.H., Galhardo, R.S., Luche, D.D., Van Sluys, M.A., Menck, C.F. and Oliva, G. (2006) Structure of the thiazole biosynthetic enzyme THI1 from Arabidopsis thaliana. J. Biol. Chem. 281, 30957-30966 https://doi.org/10.1074/jbc.M604469200

9 Hanson, A.D., Beaudoin, G.A., McCarty, D.R. and Gregory, III, J.F. (2016) Does abiotic stress cause functional B vitamin deficiency in plants? Plant Physiol. 172, 2082-2097 https://doi.org/10.1104/pp.16.01371

10 Nelson, C.J., Alexova, R., Jacoby, R.P. and Millar, A.H. (2014) Proteins with high turnover rate in barley leaves estimated by proteome analysis combined with in planta isotope labeling. Plant Physiol. 166, 91-108 https://doi.org/10.1104/pp.114.243014

11 Li, L., Nelson, C.J., Trosch, J., Castleden, I., Huang, S. and Millar, A.H. (2017) Protein degradation rate in Arabidopsis thaliana leaf growth and development. Plant Cell 29, 207-228 https://doi.org/10.1105/tpc.16.00768

12 Tivendale, N.D., Hanson, A.D., Henry, C.S., Hegeman, A.D. and Millar, A.H. (2020) Enzymes as parts in need of replacement - and how to extend their working life. Trends Plant Sci. 25, 661-669 https://doi.org/10.1016/j.tplants.2020.02.006

13 Hanson, A.D., McCarty, D.R., Henry, C.S., Xian, X., Joshi, J., Patterson, J.A. et al. (2021) The number of catalytic cycles in an enzyme's lifetime and why it matters to metabolic engineering. Proc. Natl Acad. Sci. U.S.A. 118, e2023348118 https://doi.org/10.1073/pnas.2023348118

14 Eser, B.E., Zhang, X., Chanani, P.K., Begley, T.P. and Ealick, S.E. (2016) From suicide enzyme to catalyst: the iron-dependent sulfide transfer in Methanococcus jannaschii thiamin thiazole biosynthesis. J. Am. Chem. Soc. 138, 3639-3642 https://doi.org/10.1021/jacs.6b00445

15 Sun, J., Sigler, C.L., Beaudoin, G.A.W., Joshi, J., Patterson, J.A., Cho, K.H. et al. (2019) Parts-prospecting for a high-efficiency thiamin thiazole biosynthesis pathway. Plant Physiol. 179, 958-968 https://doi.org/10.1104/pp.18.01085

16 Stewart, V. and Parales, Jr. J. (1988) Identification and expression of genes narL and narX of the nar (nitrate reductase) locus in Escherichia coli K-12. J. Bacteriol. 170, 1589-1597 https://doi.org/10.1128/jb.170.4.1589-1597.1988

17 Neidhardt, F.C., Bloch, P.L. and Smith, D.F. (1974) Culture medium for enterobacteria. J. Bacteriol. 119, 736-747 https://doi.org/10.1128/jb.119.3 736-747.1974

18 Overbeek, R., Begley, T., Butler, R.M., Choudhuri, J.V., Chuang, H.Y., Cohoon, M., et al. (2005) The subsystems approach to genome annotation and its use in the project to annotate 1000 genomes. Nucleic Acids Res. 33, 5691-5702 https://doi.org/10.1093/nar/gki866

19 Suzek, B.E., Wang, Y., Huang, H., McGarvey, P.B. and Wu, C.H. (2015) Uniref clusters: a comprehensive and scalable alternative for improving sequence similarity searches. Bioinformatics 31, 926-932 https://doi.org/10.1093/bioinformatics/btu739

20 Gerlt, J.A., Bouvier, J.T., Davidson, D.B., Imker, H.J., Sadkhin, B., Slater, D.R. et al. (2015) Enzyme function initiative-enzyme similarity tool (EFI-EST): a web tool for generating protein sequence similarity networks. Biochim. Biophys. Acta 1854, 1019-1037 https://doi.org/10.1016/j.bbapap.2015.04.015

21 Datsenko, K.A. and Wanner, B.L. (2000) One-step inactivation of chromosomal genes in Escherichia coli K-12 using PCR products. Proc. Natl Acad. Sci. U.S.A. 97, 6640-6645 https://doi.org/10.1073/pnas.120163297

22 Baba, T., Ara, T., Hasegawa, M., Takai, Y., Okumura, Y., Baba, M. et al. (2006) Construction of Escherichia coli K-12 in-frame, single-gene knockout mutants: the Keio collection. Mol. Syst. Biol. 2, 2006.0008 https://doi.org/10.1038/msb4100050 
23 Guzman, L.M., Belin, D., Carson, M.J. and Beckwith, J. (1995) Tight regulation, modulation, and high-level expression by vectors containing the arabinose $\mathrm{P}_{\text {BAD }}$ promoter. J. Bacteriol. 177, 4121-4130 https://doi.org/10.1128/jb.177.14.4121-4130.1995

24 Kabsch, W. (2010) XDS. Acta Crystallogr. D Biol. Crystallogr. 66, 125-132 https://doi.org/10.1107/S0907444909047337

25 Afonine, P.V., Grosse-Kunstleve, R.W., Echols, N., Headd, J.J., Moriarty, N.W., Mustyakimov, M. et al. (2012) Towards automated crystallographic structure refinement with phenix.refine. Acta Crystallogr. D Biol. Crystallogr. 68, 352-367 https://doi.org/10.1107/S0907444912001308

26 Emsley, P. and Cowtan, K. (2004) Coot: model-building tools for molecular graphics. Acta Crystallogr. D Biol. Crystallogr. 60, 2126-2132 https://doi. org/10.1107/S0907444904019158

27 Murshudov, G.N., Skubak, P., Lebedev, A.A., Pannu, N.S., Steiner, R.A., Nicholls, R.A. et al. (2011) REFMAC5 for the refinement of macromolecular crystal structures. Acta Crystallogr. D Biol. Crystallogr. 67, 355-367 https://doi.org/10.1107/S0907444911001314

28 Moriarty, N.W., Grosse-Kunstleve, R.W. and Adams, P.D. (2009) Electronic ligand builder and optimization workbench (eLBOW): a tool for ligand coordinate and restraint generation. Acta Crystallogr. D Biol. Crystallogr. 65, 1074-1080 https://doi.org/10.1107/S0907444909029436

29 Zallot, R., Oberg, N. and Gerlt, J.A. (2019) The EFI web resource for genomic enzymology tools: leveraging protein, genome, and metagenome databases to discover novel enzymes and metabolic pathways. Biochemistry 58, 4169-4182 https://doi.org/10.1021/acs.biochem.9b00735

30 Ettema, T.J., Huynen, M.A., de Vos, W.M. and van der Oost, J. (2003) TRASH: a novel metal-binding domain predicted to be involved in heavy-metal sensing, trafficking and resistance. Trends Biochem. Sci. 28, 170-173 https://doi.org/10.1016/S0968-0004(03)00037-9

31 Monchy, S., Benotmane, M.A., Wattiez, R., van Aelst, S., Auquier, V., Borremans, B. et al. (2006) Transcriptomic and proteomic analyses of the pMOL30-encoded copper resistance in Cupriavidus metallidurans strain CH34. Microbiology (Reading) 152, 1765-1776 https://doi.org/10.1099/mic.0. 28593-0

32 Baker-Austin, C., Dopson, M., Wexler, M., Sawers, R.G. and Bond, P.L. (2005) Molecular insight into extreme copper resistance in the extremophilic archaeon 'Ferroplasma acidarmanus' Fer1. Microbiology (Reading) 151, 2637-2646 https://doi.org/10.1099/mic.0.28076-0

33 Boyd, E.S. and Barkay, T. (2012) The mercury resistance operon: from an origin in a geothermal environment to an efficient detoxification machine. Front. Microbiol. 3, 349 https://doi.org/10.3389/fmicb.2012.00349

34 Szymanski, M.R., Fiebach, A.R., Tratschin, J.D., Gut, M., Ramanujam, V.M., Gottipati, K. et al. (2009) Zinc binding in pestivirus N(pro) is required for interferon regulatory factor 3 interaction and degradation. J. Mol. Biol. 391, 438-449 https://doi.org/10.1016/j.jmb.2009.06.040

35 Orell, A., Remonsellez, F., Arancibia, R. and Jerez, C.A. (2013) Molecular characterization of copper and cadmium resistance determinants in the biomining thermoacidophilic archaeon Sulfolobus metallicus. Archaea 2013, 289236 https://doi.org/10.1155/2013/289236

36 Zhang, Y., Rodionov, D.A., Gelfand, M.S. and Gladyshev, V.N. (2009) Comparative genomic analyses of nickel, cobalt and vitamin $B_{12}$ utilization. BMC Genomics 10, 78 https://doi.org/10.1186/1471-2164-10-78

37 Hausinger, R.P., Desguin, B., Fellner, M., Rankin, J.A. and Hu, J. (2018) Nickel-pincer nucleotide cofactor. Curr. Opin. Chem. Biol. 47, 18-23 https://doi.org/10.1016/j.cbpa.2018.06.019

38 Schuelke-Sanchez, A.E., Stone, A.A. and Liptak, M.D. (2020) Cfba promotes insertion of cobalt and nickel into ruffled tetrapyrroles in vitro. Dalton Trans. 49, 1065-1076 https://doi.org/10.1039/C9DT03601F

39 Guldan, H., Sterner, R. and Babinger, P. (2008) Identification and characterization of a bacterial glycerol-1-phosphate dehydrogenase: $\mathrm{Ni}^{2+}$-dependent AraM from Bacillus subtilis. Biochemistry 47, 7376-7384 https://doi.org/10.1021/bi8005779

40 Korshunov, S., Imlay, K.R. and Imlay, J.A. (2016) The cytochrome bd oxidase of Escherichia coli prevents respiratory inhibition by endogenous and exogenous hydrogen sulfide. Mol. Microbiol. 101, 62-77 https://doi.org/10.1111/mmi.13372

41 Hamza, M.A. and Engel, P.C. (2007) Enhancing long-term thermal stability in mesophilic glutamate dehydrogenase from Clostridium symbiosum by eliminating cysteine residues. Enzyme Microb. Technol. 41, 706-710 https://doi.org/10.1016/j.enzmictec.2007.06.008

42 Yang, H., Liu, L., Li, J., Du, G. and Chen, J. (2012) Structure-based replacement of methionine residues at the catalytic domains with serine significantly improves the oxidative stability of alkaline amylase from alkaliphilic Alkalimonas amylolytica. Biotechnol. Prog. 28, 1271-1277 https://doi. org/10.1002/btpr.1611

43 Hummel, C.S., Lancaster, K.M. and Crane, III, E.J. (2005) Determination of coenzyme A levels in Pyrococcus furiosus and other Archaea: implications for a general role for coenzyme A in thermophiles. FEMS Microbiol. Lett. 252, 229-234 https://doi.org/10.1016/j.femsle.2005.09.004

44 Rocha, E.R., Tzianabos, A.O. and Smith, C.J. (2007) Thioredoxin reductase is essential for thiol/disulfide redox control and oxidative stress survival of the anaerobe Bacteroides fragilis. J. Bacteriol. 189, 8015-8023 https://doi.org/10.1128/JB.00714-07

45 Heinemann, J., Hamerly, T., Maaty, W.S., Movahed, N., Steffens, J.D., Reeves, B.D. et al. (2014) Expanding the paradigm of thiol redox in the thermophilic root of life. Biochim. Biophys. Acta 1840, 80-85 https://doi.org/10.1016/j.bbagen.2013.08.009

46 Herrmann, J.M. and Jakob, U. (2008) Special issue: redox regulation of protein folding. Biochim. Biophys. Acta 1783, 519 https://doi.org/10.1016/j. bbamcr.2008.03.002

47 Leary, S.C. (2010) Redox regulation of SCO protein function: controlling copper at a mitochondrial crossroad. Antioxid. Redox Signal. 13, 1403-1416 https://doi.org/10.1089/ars.2010.3116

48 Vetriani, C., Speck, M.D., Ellor, S.V., Lutz, R.A. and Starovoytov, V. (2004) Thermovibrio ammonificans sp. nov., a thermophilic, chemolithotrophic, nitrate-ammonifying bacterium from deep-sea hydrothermal vents. Int. J. Syst. Evol. Microbiol. 54, 175-181 https://doi.org/10.1099/ijs.0.02781-0

49 Giovannelli, D., Sievert, S.M., Hügler, M., Markert, S., Becher, D., Schweder, T. et al. (2017) Insight into the evolution of microbial metabolism from the deep-branching bacterium, Thermovibrio ammonificans. eLife 6, e18990 https://doi.org/10.7554/eLife.18990

50 Engilberge, S., Wagner, T., Santoni, G., Breyton, C., Shima, S., Franzetti, B. et al. (2019) Protein crystal structure determination with the crystallophore, a nucleating and phasing agent. J. Appl. Crystallogr. 52, 722-731 https://doi.org/10.1107/S1600576719006381

51 Stewart, L.C., Algar, C.K., Fortunato, C.S., Larson, B.I., Vallino, J.J., Huber, J.A. et al. (2019) Fluid geochemistry, local hydrology, and metabolic activity define methanogen community size and composition in deep-sea hydrothermal vents. ISME J. 13, 1711-1721 https://doi.org/10.1038/ s41396-019-0382-3

52 Pimentel, C., Caetano, S.M., Menezes, R., Figueira, I., Santos, C.N., Ferreira, R.B. et al. (2014) Yap1 mediates tolerance to cobalt toxicity in the yeast Saccharomyces cerevisiae. Biochim. Biophys. Acta 1840, 1977-1986 https://doi.org/10.1016/j.bbagen.2014.01.032

53 Milne, N., Luttik, M.A.H., Cueto Rojas, H.F., Wahl, A., van Maris, A.J.A., Pronk, J.T. et al. (2015) Functional expression of a heterologous nickel-dependent, ATP-independent urease in Saccharomyces cerevisiae. Metab. Eng. 30, 130-140 https://doi.org/10.1016/j.ymben.2015.05.003 
54 Kalms, J., Schmidt, A., Frielingsdorf, S., van der Linden, P., von Stetten, D., Lenz, O. et al. (2016) Krypton derivatization of an $\mathrm{O}_{2}$-tolerant membrane-bound [NiFe] hydrogenase reveals a hydrophobic tunnel network for gas transport. Angew. Chem. Int. Ed. Engl. 55, 5586-5590 https://doi. org/10.1002/anie.201508976

55 Kalms, J., Schmidt, A., Frielingsdorf, S., Utesch, T., Gotthard, G., von Stetten, D. et al. (2018) Tracking the route of molecular oxygen in $\mathrm{O}_{2}$-tolerant membrane-bound [NiFe] hydrogenase. Proc. Natl Acad. Sci. U.S.A. 115, E2229-E2237 https://doi.org/10.1073/pnas.1712267115

56 Alfano, M. and Cavazza, C. (2020) Structure, function, and biosynthesis of nickel-dependent enzymes. Protein Sci. 29, 1071-1089 https://doi.org/10. 1002/pro.3836

57 Kobayashi, M. and Shimizu, S. (1999) Cobalt proteins. Eur. J. Biochem. 261, 1-9 https://doi.org/10.1046/j.1432-1327.1999.00186.x

58 Fukuzumi, S., Cho, K.B., Lee, Y.M., Hong, S. and Nam, W. (2020) Mechanistic dichotomies in redox reactions of mononuclear metal-oxygen intermediates. Chem. Soc. Rev. 49, 8988-9027 https://doi.org/10.1039/D0CS01251C

59 Wang, Y., Li, J. and Liu, A. (2017) Oxygen activation by mononuclear nonheme iron dioxygenases involved in the degradation of aromatics. J. Biol. Inorg. Chem. 22, 395-405 https://doi.org/10.1007/s00775-017-1436-5

60 Fiedler, A.T. and Fischer, A.A. (2017) Oxygen activation by mononuclear Mn, Co, and Ni centers in biology and synthetic complexes. J. Biol. Inorg. Chem. 22, 407-424 https://doi.org/10.1007/s00775-016-1402-7

61 Shearer, J., Peck, K.L., Schmitt, J.C. and Neupane, K.P. (2014) Cysteinate protonation and water hydrogen bonding at the active-site of a nickel superoxide dismutase metallopeptide-based mimic: implications for the mechanism of superoxide reduction. J. Am. Chem. Soc. 136, 16009-16022 https://doi.org/10.1021/ja5079514

62 García-García, J.D., Joshi, J., Patterson, J.A., Trujillo-Rodriguez, L., Reisch, C.R., Javanpour, A.A. et al. (2020) Potential for applying continuous directed evolution to plant enzymes: an exploratory study. Life (Basel) 10, $179 \mathrm{https://doi.org/10.3390/life10090179}$

63 Rix, G. and Liu, C.C. (2021) Systems for in vivo hypermutation: a quest for scale and depth in directed evolution. Curr. Opin. Chem. Biol. 64, 20-26 https://doi.org/10.1016/j.cbpa.2021.02.008 